\title{
Cathepsin L Promotes Vascular Intimal Hyperplasia after Arterial Injury
}

\author{
Jingjing Cai, ${ }^{1,2,3^{*}}$ Hua Zhong, ${ }^{1,2^{*}}$ Jinze Wu, ${ }^{1,2}$ Rui-Fang Chen, ${ }^{1}$ Huan Yang, ${ }^{4}$ Yousef Al-Abed, ${ }^{4}$ Ying Li ${ }^{1}$ \\ Xiaohui Li, ${ }^{1}$ Weihong Jiang, ${ }^{3}$ Marcelo F Montenegro, ${ }^{2}$ Hong Yuan, ${ }^{1,3}$ Timothy R Billiar, ${ }^{1,2}$ and Alex F Chen ${ }^{1,2,3}$ \\ ${ }^{1}$ Center for Vascular Disease and Translational Medicine, Third Xiangya Hospital, Central South University, Changsha, China; \\ ${ }^{2}$ Department of Surgery, University of Pittsburgh School of Medicine, Pittsburgh, Pennsylvania, United States of America; \\ ${ }^{3}$ Department of Cardiology, Third Xiangya Hospital, Central South University, Changsha, China; and ${ }^{4}$ The Feinstein Institute for \\ Medical Research, Manhasset, New York, United States of America
}

\begin{abstract}
The inflammatory pathways that drive the development of intimal hyperplasia $(\mathrm{IH})$ following arterial injury are not fully understood. We hypothesized that the lysosomal cysteine protease cathepsin L activates processes leading to $\mathrm{IH}$ after arterial injury. Using a mouse model of wire-induced carotid artery injury, we showed that cathepsin L activity peaks at $\mathrm{d} 7$ and remains elevated for $28 \mathrm{~d}$. Genetic deletion of cathepsin L prevented $\mathrm{H}$ and monocyte recruitment in the carotid wall. The injury-induced increases in cathepsin L mRNA and activity were mitigated in mice with myeloid-specific deletion of toll-like receptor 4 (TLR4) or myeloid differentiation primary response gene 88 (MyD88). We further discovered that the HIV protease inhibitor saquinavir (SQV), which is known to block recombinant mouse cathepsin L activity in vitro, prevented IH after arterial injury. SQV also suppressed LPS (TLR4 agonist)-induced monocyte adhesion to endothelial monolayers. These findings establish cathepsin $L$ as a critical regulator of the inflammation that leads to $\mathrm{IH}$ and that the TLR4-MyD88 pathway in myeloid lineages regulates cathepsin L expression in the vessel wall following wire injury. The Food and Drug Administration-approved drug SQV blocks IH though mechanisms that may include the suppression of cathepsin L.
\end{abstract}

Online address: http://www.molmed.org

doi: $10.2119 / \mathrm{molmed} .2016 .00222$

\section{INTRODUCTION}

Endovascular interventions for arterial occlusive disease such as angioplasty and stent placement can fail due to restenosis. These interventional procedures result in endothelial denudation with intimal and medial damage, which induces substantial local inflammation. This inflammation is manifested by monocyte infiltration as well as inflammatory mediator and growth factor production (1). This in turn stimulates vascular smooth muscle cell (SMC) accumulation and extracellular matrix deposition, resulting in intimal hyperplasia (IH) and vessel or stent occlusion (2-4). Proteases contribute to IH and vascular remodeling by degrading matrix components and regulating inflammatory cell infiltration (5).

Cysteine cathepsins are lysosomal proteases that can be released by cells or expressed on the surface of cells (6). Cathepsin expression is highest in macrophages, where they serve to degrade

*JC and HZ contributed equally.

Address correspondence to Timothy R Billiar, UPMC Presbyterian Hospital, 200 Lothrop Street, Suite F1281, Pittsburgh, PA 15213. Phone: 412-647-1749; Fax: 412-647-3247;

E-mail: billiartr@upmc.edu. Alex F Chen, W939 Biomedical Science Tower, Department of Surgery, University of Pittsburgh School of Medicine, 200 Lothrop Street, Pittsburgh, PA 15213. Phone: 412-624-7784; Fax: 412-383-5946; E-mail: afychen@yahoo.com. Submitted November 12, 2016; Accepted for Publication March 15, 2017; Published Online (www.molmed.org) March 20, 2017.

YY:

Veinstein Institute

for Medical Research

Northwell Health

proteins in the lysosomes and participate in extracellular matrix remodeling when released by cells (6). Cathepsin $\mathrm{L}$ has been shown to regulate neovascularization in the heart $(7,8)$ and aneurysm formation (9). There are two distinct cathepsin L proteases encoded by the human cathepsin L gene, namely cathepsin L1 and cathepsin V (L2). Mouse cathepsin L is most homologous to human cathepsin V $(10,11)$. Since macrophage infiltration and activation are critical steps in the $\mathrm{IH}$ that follows acute injury to the arterial wall (12-14), we postulate that cathepsin L contributes to IH and that the TLR4MyD88 pathway in myeloid lineages regulates cathepsin $\mathrm{L}$ expression after wire injury.

We (12) and others (15-17) have shown that TLR4/MyD88 signaling is significantly involved in IH after arterial injury. We show here that cathepsin L expression is markedly increased after carotid artery wire injury and that this depends 
on TLR4/MyD88 signaling in myeloid cells. Using cathepsin $\mathrm{L}^{-/-}$mice, we also show that cathepsin $\mathrm{L}$ is involved in $\mathrm{IH}$. Finally, we show that a Food and Drug Administration-approved protease inhibitor, saquinavir (SQV), known to inhibit cathepsin L, significantly blocks IH after arterial injury. Therefore, cathep$\sin \mathrm{L}$ could represent a novel target to prevent IH.

\section{MATERIALS AND METHODS}

Antibodies against CD68 (ab955), MyD88 (SAB2500664), $\beta$-actin (A1978), anti-actin and $\alpha$-smooth muscle-Cy3 (C6198) were purchased from Sigma. Antibody against cathepsin L (ab133641) and cathepsin L Activity Assay Kits (Fluorometric) (ab65306) were purchased from Abcam. Antibody against cathepsin L (AF1515) was purchased from R\&D. Saquinavir and ritonavir were provided by Dr. Yousef Al-Abed (The Feinstein Institute for Medical Research). In the in vivo study, mice were given saquinavir $5 \mathrm{mg} / \mathrm{kg}$ and ritonavir $1.25 \mathrm{mg} / \mathrm{kg}$ intraperitoneally once daily $3 \mathrm{~d}$ before surgery and $28 \mathrm{~d}$ after surgery.

\section{Animals}

MyD88 ${ }^{\mathrm{loxP} /} \mathrm{L}^{\text {oxP }}$ mice, LyzM-cre

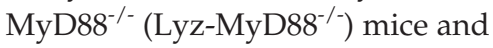
cathepsin $\mathrm{L}^{-/-}$mice were generated or bred in the surgery department of the University of Pittsburgh School of Medicine (18). All mutant strains were backcrossed at least 6 times onto a C57BL/ 6 background and used at the age of 8-12 wks. Animals were maintained on a $12 \mathrm{~h}$ light-dark cycle in a pathogen-free facility at the University of Pittsburgh and were fed a standard diet with water.

All animal protocols and experimental designs were approved by the University of Pittsburgh Institutional Animal Care and Use Committee and were in accordance with the guidelines provided by the National Institute of Health and the International Association for the Study of Pain. The origins of all mutant mice used are listed in Supplementary Table S1.

\section{Carotid Artery Injury Model}

Carotid artery wire injury was induced just proximal to the carotid bifurcation as previously described (19). Briefly, mice were anesthetized with isoflurane and the left carotid artery was exposed on the ventral side of the neck via a midline incision. The bifurcation of the right carotid artery was located, and two ligatures (surgical silk, size 6-0; Deknatel) were placed around the external carotid artery, which was then tied off with the distal ligature. After temporary occlusion of the internal carotid artery, a small incision was made between the two ligatures placed around the external carotid artery, and a curved, straight guide wire (C-SF-15-15, Cook Belgium) was passed toward the aortic arch and withdrawn three times with a rotating motion to denude endothelial cells. Ligation of the external carotid artery was done after withdrawal of the guide wire to increase the neointimal area (20). The skin incision was closed with a single suture. Animals were allowed to recover and carotid arteries were harvested $28 \mathrm{~d}$ after the injury. In the sham operation group, the left carotid artery was exposed on the ventral side of the neck via a midline incision. The vessels were not cannulated or injured and the incision was closed. Data are from four to eight mice per group in each experiment. Carotid common arteries (from the bifurcation to the aortic arch) were used for enzymatic activity analysis and western blot. The middle section of the carotid common artery was used for immunofluorescence staining. The number and mortality of mice in each group are given in the figure legends and Supplementary Table S1.

\section{Histological and Morphometric Analysis}

Carotid arteries were harvested at the indicated times after injury. Five to six $6 \mu \mathrm{m}$ thick sections, cut $100 \mu \mathrm{m}$ apart from each other, were stained with hematoxylin and eosin-stained. The five or six sections obtained from each arterial segment were analyzed with computerized morphometry (ImageJ software, National Institutes of Health), and the average values were calculated. Measurements included luminal area, medial area, intimal area, vessel area, and the lengths of the internal elastic lamina and external elastic lamina. The intima-to-media (I/M) ratio was calculated as previously described (12). All sections were analyzed separately by two investigators blinded to the study design. Immunohistochemical analyses were processed according to standard procedures.

\section{Immunofluorescent Staining}

Carotid arteries were harvested at the indicated times after injury and were fixed in $2 \%$ paraformaldehyde for $2 \mathrm{~h}$. Tissue was placed in 30\% sucrose for $24 \mathrm{~h}$. Cryostat sections $(6 \mu \mathrm{m})$ were obtained and put onto gelatin-coated glass slides permeabilized with $0.1 \%$ Triton X-100 for $10 \mathrm{~min}$ and blocked with $2 \%$ bovine serum albumin for $45 \mathrm{~min}$. Tissues were then incubated in primary antibodies overnight at $4^{\circ} \mathrm{C}$, followed by incubation for $60 \mathrm{~min}$ with fluorescent-labeled secondary antibodies. After nuclear staining for $30 \mathrm{~s}$ with 6-diamidino-2-phenylindole (DAPI), slides were covered using Gelvatol. Antibody dilutions were prepared as follows: cathepsin L, 1:100; CD68, 1:100; secondary donkey anti-rabbit (488) Alexa Fluor-conjugated antibodies, 1:500; and 4', DAPI, 1:2000. Images were taken using an Olympus Provis fluorescence microscope or an Olympus Fluoview 500 confocal microscope at the University of Pittsburgh Center for Biologic Imaging. Quantitative analyses of immunohistochemistry experiments were performed on digitized images $(10 \times$ primary magnification $)$ by using Photoshop software. The presentative sections at the level of the injured carotid artery were taken from each animal (three to five animals per genotype). To count CD68-positive cells in the carotid artery, four squared counting boxes (75 $\mu \mathrm{m}$ per side) were taken per section. 


\section{Reverse Transcription-Quantitative Polymerase Chain Reaction}

RNA from carotid artery tissue was isolated using the RNeasy Mini Kit (Qiagen). Reverse-transcription into cDNA and quantitative polymerase chain reactions (PCRs) were carried out using iScript ${ }^{\mathrm{TM}}$ One-Step RT-PCR Kit With SYBR® Green (Bio-Rad). The reaction was run on a CFX96 ${ }^{\mathrm{TM}}$ real-time PCR detection system. PCR primers listed in Supplementary Table S2 were used as an internal control, and the data are expressed as -fold of a corresponding control.

\section{Western Blot Analysis}

Whole cell lysates were collected in CelLytic ${ }^{\text {TM }}$ MT Cell Lysis Reagent (Sigma Aldrich) and protease inhibitor cocktail (Sigma Aldrich). Protein concentrations were determined by the micro bicinchoninic acid assay (Pierce). Equal amounts of lysates (30 $\mu$ g protein) were separated by SDS-PAGE and transferred to a nitrocellulose membrane that was blocked with Odyssey blocking buffer (LI-COR) for $60 \mathrm{~min}$, followed by incubation overnight at $4^{\circ} \mathrm{C}$ with an anti- $\beta$ actin (1:2000) and anti-cathepsin L (1:1000), and then washed before incubation with species-appropriate, fluorescently conjugated secondary antibodies for $1 \mathrm{~h}$ at room temperature. Membranes were analyzed using an Odyssey Infrared Imaging System (LI-COR) and relevant signal intensity was determined using Image J software. Significance was established using Student $t$ test; a $p$ value of $<0.05$ was considered significant.

\section{Cathepsin L Activity Assay}

Carotid artery tissue cathepsin L activity was measured using a cathepsin L Activity Assay Kit (Fluorometric) from Abcam, following the manufacturer's protocol. Briefly, whole tissue lysates from carotid arteries were collected in $100 \mu \mathrm{l}$ of CL cell lysis buffer. Cells were lysed in $100 \mu$ of chilled CL cell lysis buffer. Tissue was incubated on ice for $10 \mathrm{~min}$. Whole cell lysates were collected and protein concentrations determined by the micro bicinchoninic acid assay (Pierce), then $50 \mu \mathrm{l}$ of tissue lysates were added to a 96-well plate and $50 \mu \mathrm{l}$ of CL reaction buffer was added to each sample and $2 \mu \mathrm{l}$ of the $10 \mathrm{mM}$ AcFR-AFC substrate was added to each well. These were incubated at $37^{\circ} \mathrm{C}$ for $1-2 \mathrm{~h}$. Each sample was read by a bottom-reading fluorometer at excitation/emission wavelengths of 400/505 nm. Arterial enzyme activity for cathepsin $\mathrm{L}$ was normalized to tissue extract protein concentration at indicated time points following wire injury.

\section{Endothelial Cell Adhesion Assays}

Endothelial cell adhesion assays were performed using a commercially available kit (ECM645, Millipore). Briefly, human umbilical vein endothelial cells (HUVEC) were seeded in black fluorescence tissue culture plates and grown for $48 \mathrm{~h}$ or until confluence occurred. THP-1 cells were treated with control or LPS 10ng/mL, then calcein AM-labeled THP-1 cells were added to the wells. The plate was incubated for $2 \mathrm{~h}$ to allow for THP-1 adhesion to the endothelial monolayers, followed by rinsing with media to remove non-adherent cells. Finally, the plate was read at $485 \mathrm{~nm} / 530 \mathrm{~nm}$ wavelengths in a fluorescence plate reader.

\section{Statistical Analysis}

All data are expressed as mean \pm standard error of the mean (SEM). One-way analysis of variance following post hoc Student-Newman-Keuls test was used to determine the differences among multiple groups. The Mann-Whitney U test was applied on small-size comparisons with non-normal distributions between groups. $T$ test was applied only on experiments with normal distributions between the comparison groups. A $p$ value $<0.05$ was considered statistically significant.

All supplementary materials are available online at www.molmed.org.

\section{RESULTS}

\section{Cathepsin L Drives Intimal Hyperplasia and Macrophage Infiltration in a Carotid Artery Wire Injury Model}

Since macrophages express high levels of cathepsins, and because we (12) and others $(1,13,14)$ have shown that macrophages infiltrate carotid arteries early following wire injury, we hypothesized that cathepsin L activity would increase in the arterial wall after injury. As shown in Figure 1A, wire injury to the carotid artery in mice induced an increase in cathepsin L activity that first became evident at $3 \mathrm{~d}$, peaked at $7 \mathrm{~d}$, and remained elevated to the $28 \mathrm{~d}$ final time period studied. Cathepsin L mRNA levels were measured at the same time points. The data indicated that corresponding increases in cathepsin L mRNA levels correlated with the enzymatic activity after acute wire injury (Supplementary Figure S5). We tested whether cathepsin L was involved in injury-induced $\mathrm{IH}$ and vascular remodeling in the carotid artery wire injury model using cathepsin $\mathrm{L}^{-/-}$ mice. A marked reduction in IH and vascular remodeling was observed in injured carotid arteries from cathepsin $\mathrm{L}^{-/-}$mice $(p<0.01)$ compared with vessels from wild-type mice. Deletion of cathepsin L significantly suppressed media area by $42.2 \%$ (Figure 1B), I/M ratio by $40.7 \%$ (Figure 1C) and intimal area by $73 \%$ (Supplementary Figure S3). Cathepsin L deletion also significantly suppressed $\mathrm{CD}^{+} 8^{+}$cell infiltration by $43.9 \%$ at $\mathrm{d} 3$ after carotid artery injury (Figure 1D). Thus, cathepsin $\mathrm{L}$ regulates the processes leading to $\mathrm{IH}$, including the accumulation of monocytes/macrophages.

\section{The TLR4/MyD88 Pathway Mediates Cathepsin L Upregulation in the Vessel Wall Following Wire Injury}

We have previously shown that TLR4 on myeloid cells plays a key role in the development of $\mathrm{IH}$ in the carotid artery wire injury model (12). Here, we show that myeloid-specific deletion of TLR4 or its downstream signaling adapter protein, MyD88, significantly prevented the increases in cathepsin $\mathrm{L}$ mRNA levels observed $3 \mathrm{~d}$ after wire injury (Figures 2A, B, C). Although we had previously shown that global MyD88 deletion suppresses intimal hyperplasia (12), we now show that myeloid-specific MyD88 deletion also 

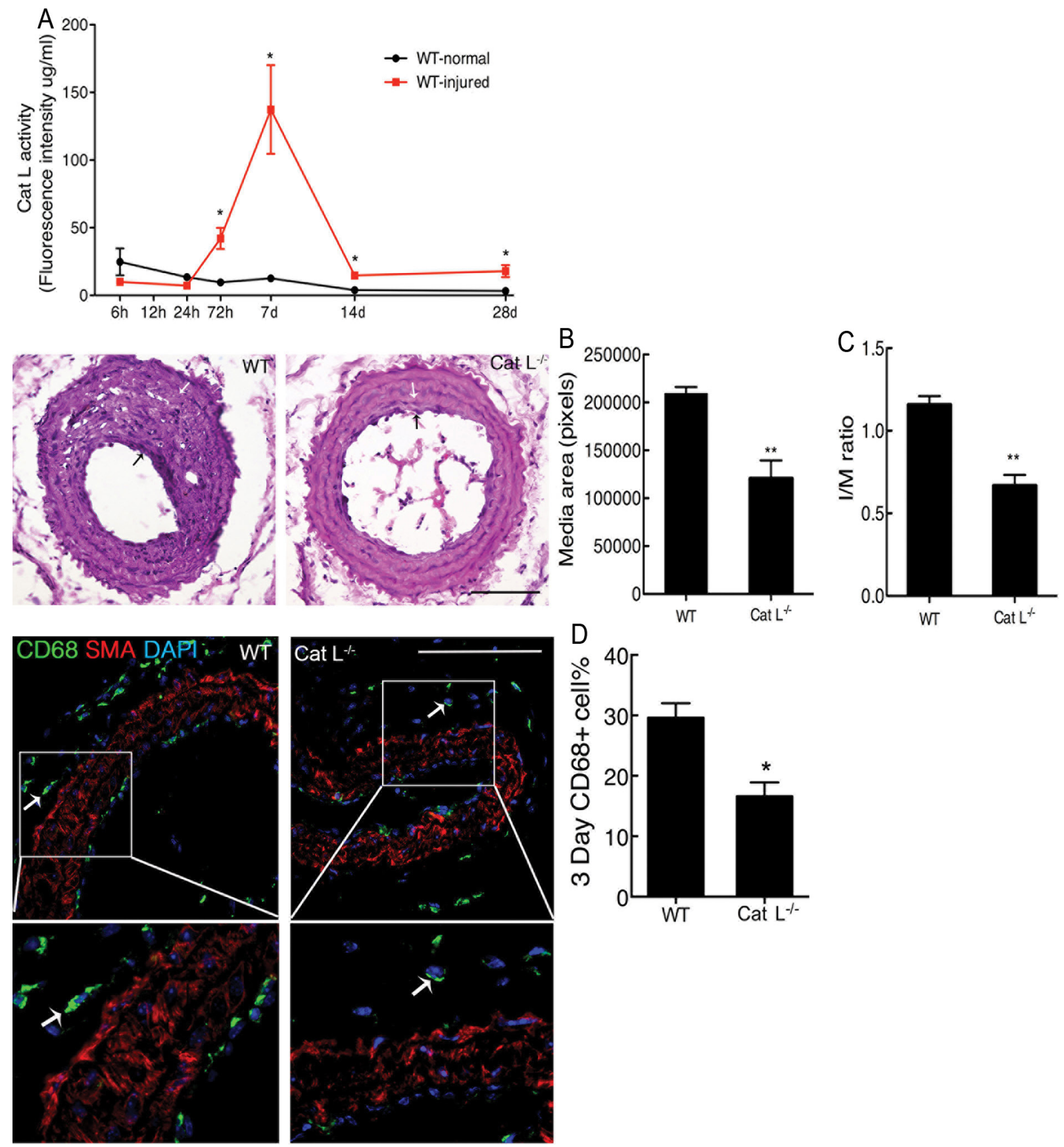

Figure 1. Cathepsin $L$ drives intimal hyperplasia and macrophage infiltration. (A) Arterial enzyme activity for cathepsin $L$ was measured by a fluorescence-based assay and normalized to tissue extract protein concentration at indicated time points following wire injury. Cathepsin L activity of the injured and uninjured carotid arteries from wild-type mice are displayed ( $\mathrm{n}=4$ at each time point).

$(B, C)$ Common carotid arteries were injured by a $0.4 \mathrm{~mm}$ guide wire plus external carotid artery ligation and harvested $28 \mathrm{~d}$ after surgery. Frozen tissues were sectioned. Representative hematoxylin and eosin stained sections of carotid arteries from wild-type mice $(n=6)$ and cathepsin $\mathrm{L}^{-1-}$ mice $(n=6)$ are shown. The ratios of intima and media area and vessel medial size were quantified by planimetry at $28 \mathrm{~d}$. The white arrows indicate internal elastic lamina. The black arrows indicate luminal side of the artery. (D) Mouse carotid arteries that were immunostained for macrophage markers (CD68-positive cells) $3 \mathrm{~d}$ after injury. The number of CD68-positive cells was defined as the percentage of total cells within a given area that stained positive for CD68. Representative hematoxylin and eosin stained and immunofluorescent-stained sections are shown. Data represent the means \pm SEM. ${ }^{*} P<0.05,{ }^{* *} P<0.01$ versus respectively controls. Scale bar represents $100 \mu \mathrm{m}$. 

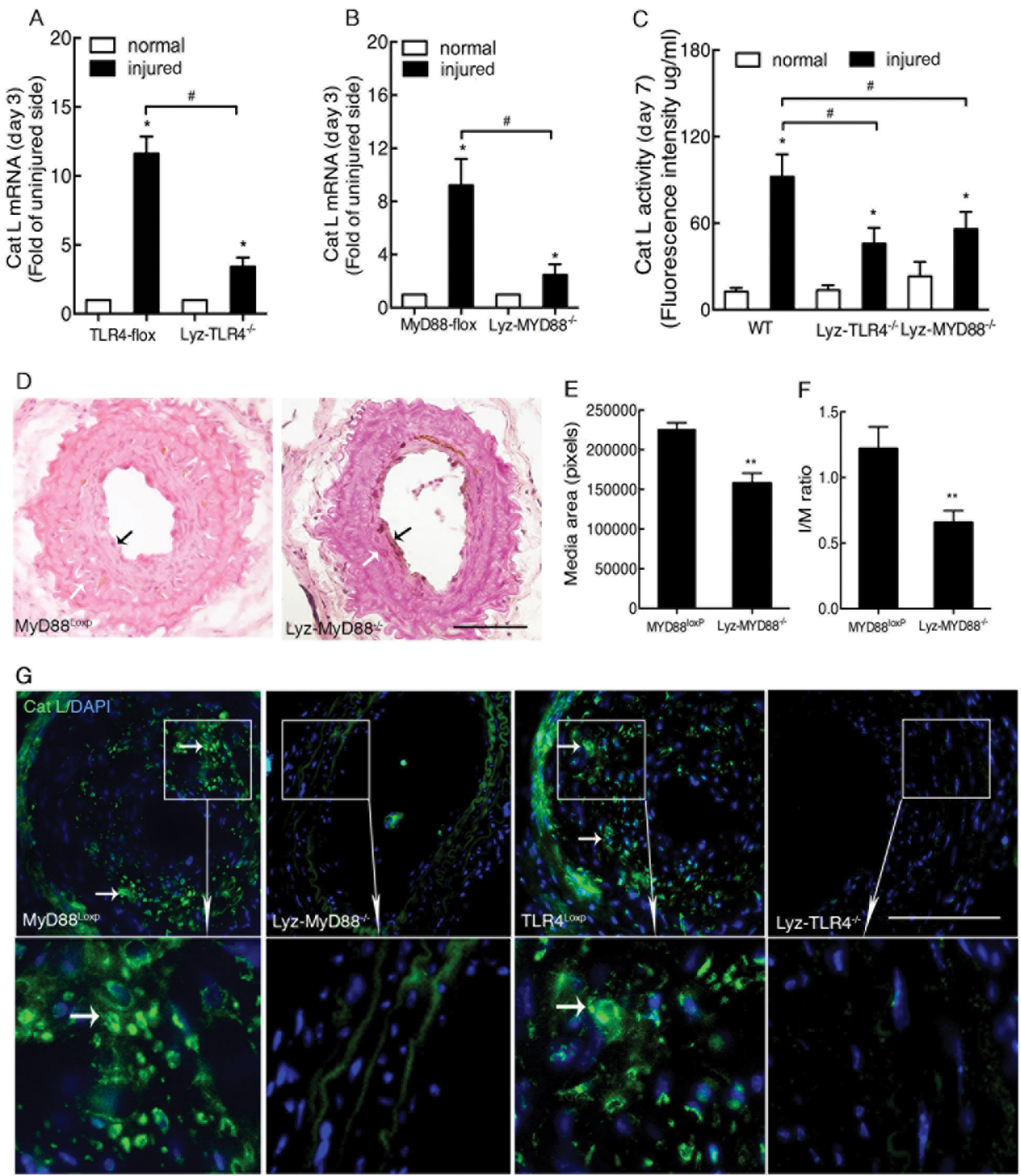

Figure 2. TLR4- MyD88 signaling in macrophage may regulate cathepsin $L$ activity and vascular remodeling. Common carotid arteries were injured by a $0.4 \mathrm{~mm}$ guide wire plus external carotid artery ligation. $(A, B)$ Arterial mRNA levels for cathepsin $L$ was measured by reverse transcription polymerase chain reaction and normalized to $18 \mathrm{~S}$ transcript levels $3 \mathrm{~d}$ following wire injury. Relative mRNA levels compared with the uninjured carotid artery are displayed $(n=4-6)$. (C) Arterial enzyme activity for cathepsin $L$ was measured by a fluorescence-based assay and normalized to tissue extract protein concentration at $d 7$ following wire injury. Cathepsin $L$ activity of the injured and uninjured carotid arteries from wild-type, Lyz-TLR4 ${ }^{-/-}$and Lyz-MyD88 ${ }^{-/-}$mice are displayed $(n=4$ at each time point).

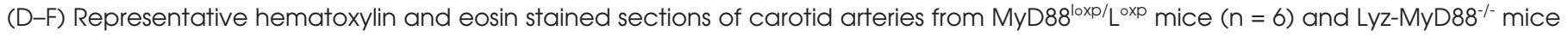
$(n=8)$ are shown. The ratios of intima and media area and vessel medial size were quantified by planimetry at $28 \mathrm{~d}$. The white arrows indicate internal elastic lamina. The black arrows indicate luminal side of the artery. $(G)$ Carotid arteries at $28 \mathrm{~d}$ after injury that were immunostained for mouse cathepsin $\mathrm{L}$. The arrows indicate cells stained positive for cathepsin $\mathrm{L}$. Representative hematoxylin and eosin stained and immunofluorescent-stained sections are shown. Data represent the means $\pm \mathrm{SEM} .{ }^{*} P<0.05,{ }^{* *} P<0.01$ versus respectively controls. Scale bar represents $100 \mu \mathrm{m}$. 
reduces intimal area, medial thickening and I/M ratio following wire injury to a level similar to that seen after TLR4 myeloid-specific knockout (Figures 2D, E, F and Supplementary Figure S3). The number of cathepsin L-positive cells in the intima was also significantly lower in the myeloid-specific TLR4 or MyD88 knockout mice compared with the TLR4 $4^{\text {loxp} /} \mathrm{L}^{\text {oxp }}$ or MyD88 $8^{\text {loxp} /} \mathrm{L}^{\text {oxp }}$ control strains at $28 \mathrm{~d}$ after wire injury (Figure 2G). Thus, the increases in cathepsin L expression at the mRNA and protein levels in the vessel wall after arterial injury involves TLR4/ MyD88 signaling in myeloid cells.

\section{Saquinavir Inhibits Cathepsin L Activity and Intimal Hyperplasia Following Wire Injury to the Carotid Artery}

We have recently shown that the first-generation HIV protease inhibitor saquinavir (SQV) blocks cathepsin L activity and suppresses high mobility group box-1 (HMGB1)-induced TLR4 signaling in macrophages (21). Because our previous work also suggested that HMGB1-induced TLR4 activation contributes to IH through TLR4 signaling on macrophages (12), we postulated that SQV would suppress wire injuryinduced IH. As shown in Figures 3A, B, C and Supplementary Figure S3, daily SQV treatment at a dose of $5 \mathrm{mg} / \mathrm{kg}$ almost completely blocked the intimal area, medial thickening and I/M ratio in the carotid wire injury model. SQV treatment was compared with mice that received the vehicle (DMSO) at equivalent amounts. We confirmed that SQV treatment prevented increases in cathepsin L activity compared with the control vehicle-treated mice following wire injury (Figure 3D). We also assessed the levels of the 26KD cleavage fragment of cathepsin L following wire injury (Figures 3E, F). Injury in the vehicle-treated mice resulted in an increase in the 26KD fragment. This was inhibited in the SQV-treated mice, indicating that SQV blocked cathepsin $\mathrm{L}$ activation in the vessel wall.

\section{Mechanistic Studies on the Role of Cathepsin L on IH after Wire Injury}

An early step in monocyte recruitment into the injured vessel wall is thought to involve adhesion of monocytes. To assess the importance of cathepsin $\mathrm{L}$ in monocyte adhesion, we measured the adhesion of cultured THP-1 monocytes to endothelial monolayers. As shown in Figure 3G, the TLR4 agonist LPS induced a significant increase in monocyte adhesion that was suppressed by both SQV and the known selective cathepsin L inhibitor SID26681509. These data suggest that cathepsin L contributes to adhesion of monocytes to the endothelium following activation of TLR4. Thus, SQV could block $\mathrm{IH}$, in part, by preventing monocyte adhesion to the vessel wall at the site of injury.

Mouse cathepsin L is homologous to human cathepsin V (also known as cathepsin L2). To determine if extracellular cathepsin $\mathrm{V}$ could regulate human aortic smooth muscle cell (HASMC) proliferation in vitro, HASMCs were exposed to recombinant cathepsin $\mathrm{V}$ at $1 \mathrm{ng} / \mathrm{mL}$ and $10 \mathrm{ng} / \mathrm{mL}$ at $\mathrm{pH} 7.4$ or 4.0. The acidic $\mathrm{pH}$ was included because cathepsins are known to be enzymatically active at low $\mathrm{pH}$ (Supplementary Figure S4A). No effect of cathepsin L on HASMC proliferation was seen after $48 \mathrm{~h}$ (Supplementary Figure S4B).

\section{DISCUSSION}

Formation of IH after arterial injury involves activation of TLR4/MyD88 signaling on myeloid cells, including macrophages (12). The damage-associated molecular pattern molecule, HMGB1, is one of the endogenous activators of TLR4 in this setting. We show here that the upregulation of the cysteine protease cathepsin L is dependent on TLR4/ MyD88 signaling in myeloid cells early after injury to the arterial wall. We also show that a recently described inhibitor of cathepsin L, the first-generation HIV protease inhibitor SQV, blocks IH after wire injury to the carotid. These findings indicate that cathepsin L is importantly involved in $\mathrm{IH}$ and could be a new target to prevent IH (Figure 4).
Cysteine cathepsins B, L and S, which are endosomal and lysosomal proteases, participate in numerous physiological systems and are upregulated during inflammatory disorders and cancers. In humans, cathepsins can regulate apoptosis, major histocompatibility complex class II-dependent immune responses, antigen processing and extracellular matrix remodeling (22-24). Two distinct cathepsin L proteases are encoded by human cathepsin L gene, namely cathepsin L1 and cathepsin V (also known as cathepsin L2). Cathepsin L1 has no homologue in the mouse. Mouse cathepsin L is homologous to human cathepsin $\mathrm{V}$ based on its amino acid sequence homology and expression pattern (9). Therefore, translation of our results into humans would focus on the role of human cathepsin V $(9,10,25)$. Macrophages have the highest cathepsin expression. Approximately $60 \%$ of the total elastolytic activity of human macrophages can be attributed to cysteine proteases, with cathepsins $\mathrm{V}, \mathrm{K}$ and $\mathrm{S}$ contributing equally. About two-thirds of cathepsin activity is found to occur extracellularly and one-third intracellularly in human cells $(10,11)$. Our results show that cathepsin L activity was increased by $72 \mathrm{~h}$ and through $28 \mathrm{~d}$ following the arterial injury, peaking at $\mathrm{d} 7$. Deletion of cathepsin L significantly attenuated IH and inflammatory cell infiltration. The role of cathepsin L in neovascularization, coronary artery disease and aneurysm formation has been established $(8,23)$. Based on our in vitro results showing that inhibition of cathepsin L blocked monocyte adhesion to endothelial cells, we speculate that monocyte cathepsin L is critical for adhesion and infiltration of those cells into the arterial wall. This role of cathepsin L might also be important for the observation that cathepsin $\mathrm{L}$ contributes to destabilization of human atherosclerotic plaques $(26,27)$. Whether the higher expression of cathepsins in atherosclerotic plaques could contribute to the exaggerated $\mathrm{IH}$ response seen in models of atherosclerosis is an interesting possibility. 
A
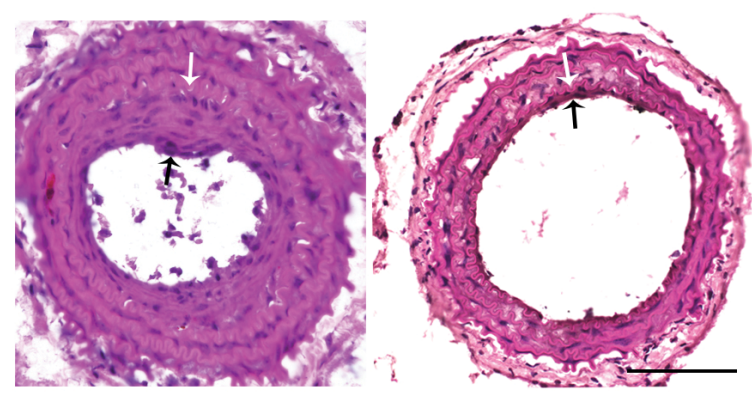

D

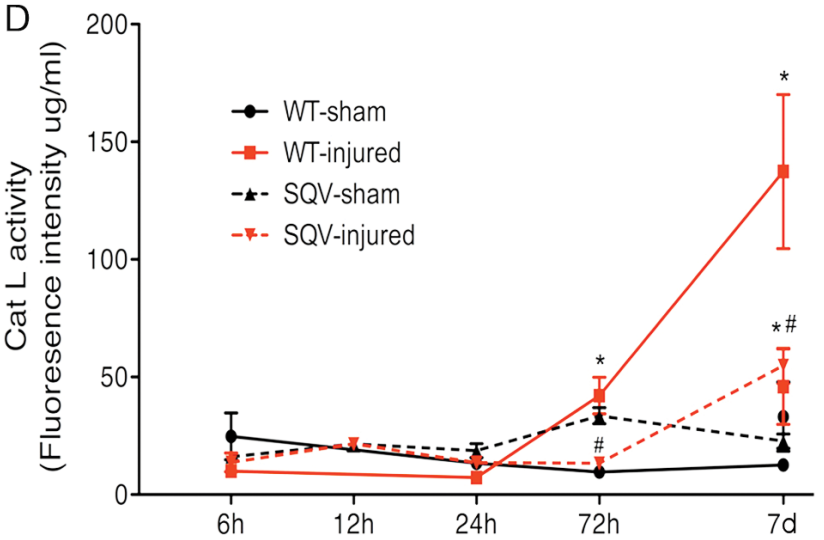

G

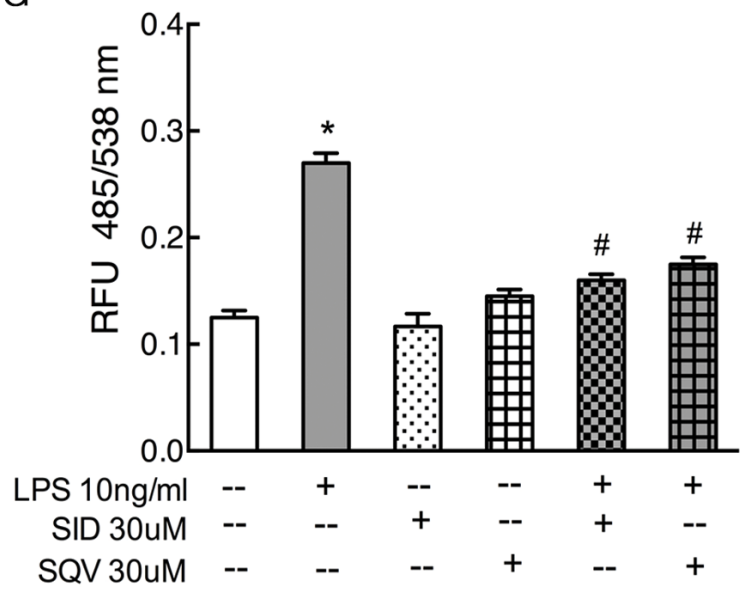

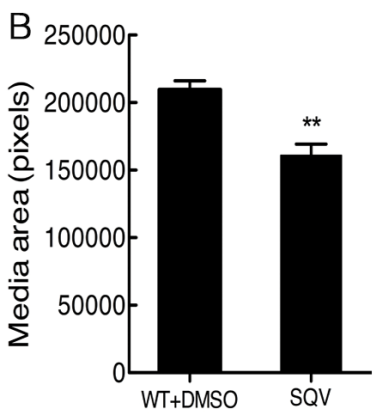

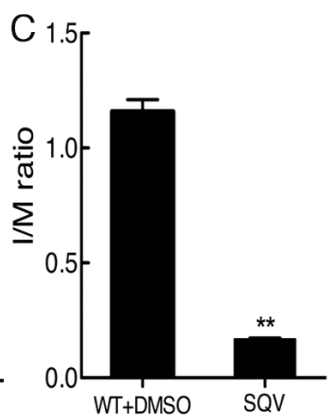

$\mathrm{E}$ Day 7

$\mathrm{F}$

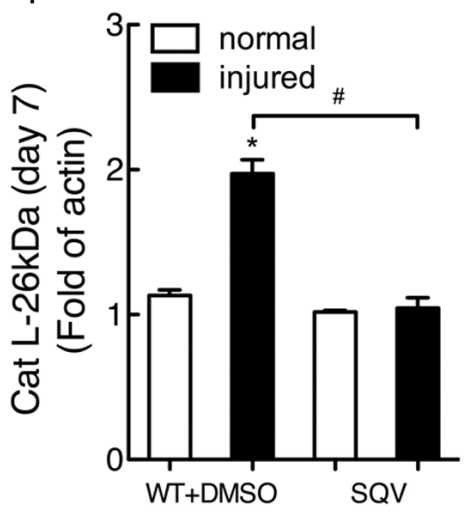

Figure 3. Saquinavir inhibits cathepsin L activity and intimal hyperplasia. (A-C) Administration of $S Q V$ ameliorates intimal hyperplasia in wild-type mice. SQV (saquinavir $5 \mathrm{mg} / \mathrm{kg}$ and ritonavir $1.25 \mathrm{mg} / \mathrm{kg}$ ) or DSMO control was administered intraperitoneally once daily $3 \mathrm{~d}$ before surgery and $28 \mathrm{~d}$ after surgery. The ratios of intima and media area and vessel medial size were quantified by planimetry at $28 \mathrm{~d}$. Representative hematoxylin and eosin stained sections of carotid arteries from control mice $(n=7)$ and SQV-treated mice $(n=8)$ are shown. The white arrows indicate internal elastic lamina. The black arrows indicate luminal side of the artery. (D) Arterial enzyme activity for cathepsin $L$ was measured by a fluorescence-based assay and normalized to tissue extract protein concentration at indicated time points following wire injury. Cathepsin L activity of the injured and the uninjured carotid arteries from control and SQV-treated mice are displayed ( $n=4$ at each time point). ${ }^{*} P<0.05$ versus sham group. $\# P<0.05$ versus the wild-type injured group. (E,F) Tissue lysates from aortic arteries $(30 \mu \mathrm{g})$ were prepared from DMSO control and $\mathrm{SQV}$-treated mice at $7 \mathrm{~d}$ after injury and subjected to western blot assay for cathepsin $L(n=4)$. ${ }^{*} P<0.05$ versus sham group. $\# P<0.05$ versus the wild-type injured group. (G) Quantification of THP- 1 cell adhesion to HUVEC. THP- 1 with treatment as indicated was labeled with the calcein AM and added to confluent monolayers of HUVEC. The plate was read at $485 / 538 \mathrm{~nm}$ in a fluorescent plate reader. Quantitative analysis of binding of THP- 1 cells to HUVEC is presented with bar graphs of fluorescence values. Data represent the means \pm SEM. ${ }^{*} P<0.05$ versus control. $\# P<0.05$ versus LPS group. Scale bar represents $100 \mu \mathrm{m}$. 


\section{Vascular Injury-Induced Inflammation}

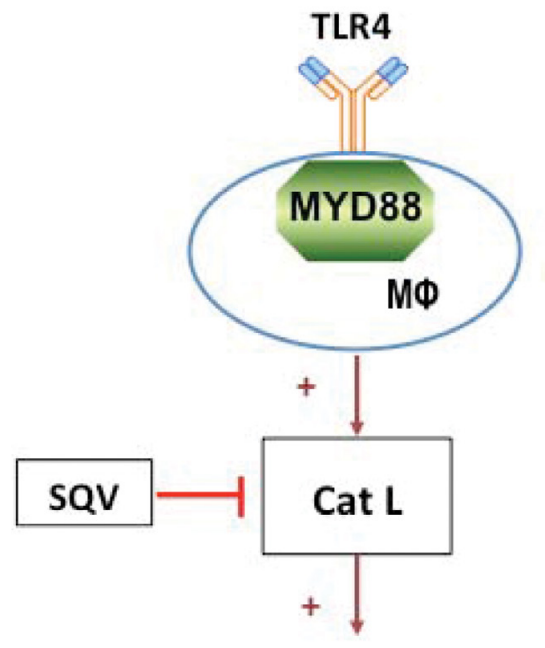

Intimal Hyperplasia

Figure 4. An illustration of potential mechanisms in injury-induced vascular intimal hyperplasia is depicted. We propose that $\mathrm{IH}$ is driven, in part, by TLR4 -MyD88 signaling on macrophages and cathepsin L pathway responding to injured and stressed cells in the vessel wall. SQV can be a potential therapeutic drug to inhibit cathepsin L activity and $\mathrm{H}$. For detailed discussion of the pathways, please see the text. ( + indicates stimulatory effect;

- I indicates inhibitory effect)

Our previous study and current data show that selective deletion of TLR4 or MyD88 on myeloid lineages abolished the upregulation of cathepsin L activity, media remodeling and $\mathrm{IH}$. Therefore, it is likely that TLR4-MyD88 signaling in macrophage is a critical regulator of cathepsin L activity. In this study, we could not conclude that cathepsin L in macrophage is the only cause of IH. Macrophages are likely to be among the sources of cathepsin L that significantly contribute to IH. Cathepsin L in different cell types may be involved in the pathophysiology of IH. This possibility will need to be further investigated in future studies (Supplementary Figure S1-S2). Vascular sterile inflammation is recognized as a pathogenic process in developing vascular IH (12). Infiltration of inflammatory cells including monocytes/macrophages is the initial event of the vascular inflammatory response (28). Some essential proinflammatory mediators, such as cytokines, chemokines and adhesion molecules, recruit inflammatory cells into the vascular wall and propagate the inflammatory response for the formation of IH. TLR4 activation by endogenous agonist like HMGB1, released by injured or necrotic cells, has been implicated in the pathogenesis of injury-driven disorders such as atherosclerosis and restenosis (12,28-30). The TLR4/MD2 receptor complex utilizes the adapter molecule MyD88 and TIR domain-containing adapter-inducing interferon- $\beta$ signaling adapters to induce expression of cytokines and chemokines (31). Previous studies implicated MyD88 in IH formation following periadventitial cuff placement on the carotid artery (15) and the inward remodeling induced by the changes of hemodynamic forces on conduit arteries (17). We showed that TLR4/MyD88 signaling is a major pathway of the arterial response to endoluminal wire injury (12) and now provide further evidence that TLR4 and MyD88 in myeloid cells are critical to the response through the upregulation of cathepsin L. Tissue resident macrophages are a heterogeneous population of immune cells that differentiate in tissuespecific niches. Evidence has been presented to indicate that cathepsins are expressed in a tissue-specific fashion. This has been linked to proteolysis of specific molecular targets as part of antigen processing/presenting during the differentiation of specific macrophage subpopulations (2-4). Whether tissue-specific cathepsin L expression is part of the arterial injury response is not known.

Vascular smooth muscle cells (VSMC) play an important role in regulating vessel wall remodeling during atherosclerosis and restenosis. To address the possibility that cathepsin could alter VSMC phenotype, we exposed HASMC to recombinant human cathepsin $\mathrm{V}$ (homologous to mouse cathepsin L) at neutral and acidic $\mathrm{pH}$. We could not find evidence that extracellular cathepsin V regulates VSMC proliferation. Previous studies showed that an increase in autophagy is essential for the conversion of VSMC from a contractile to a synthetic phenotype during vascular injury (5). Impairment of autophagy can result in alterations in the responses of VSMC and contribute to disease progression during atherosclerosis and restenosis. Cathepsins reside in lysosomes and are critical proteolytic enzymes responsible for degradation processes in the autophagosome. Recent studies have shown that cathepsin L deficiency impairs the turnover of autophagolysosomes. Therefore, one possible explanation for the reduced $\mathrm{IH}$ in cathepsin knockout mice is alterations in autophagy (6-8).

SQV acts primarily as peptidomimetic inhibiting the HIV protease. Recent reports show that SQV inhibits vascular remodeling in pulmonary artery hypertension and inflammation in nephritis (32-37). Metalloproteinases 2 and $20 S$ and $26 \mathrm{~S}$ proteasomes are proposed protease targets in mammalian tissues (32-37). However, the targets for HIV protease inhibitors after acute vascular injury have not been defined. A recent paper using a cell-based screening assay identified the mammalian protease cathepsin $\mathrm{V}$ (homologous to mouse cathepsin L) as a novel therapeutic target to inhibit TLR4-mediated inflammation induced by extracellular HMGB1 (21). SQV inhibits the interaction between cathepsin V and the TLR4-MyD88 receptor complex. We have also previously shown that HMGB1 contributes to IH in the wire injury model through TLR4 signaling (12). Because SQV is known to also block human cathepsin $\mathrm{V}$ activity, our results raise the possibility that SQV could be useful in limiting vascular remodeling responses in humans. However, this would require that human cathepsin $\mathrm{V}$ carries out roles similar to what we have shown here for mouse cathepsin $\mathrm{L}$ in the IH response. Since SQV is an FDAapproved drug with a well-established safety profile, it could be used as a therapy to prevent $\mathrm{IH}$. 


\section{ACKNOWLEDGMENTS}

This work was supported in part by the National Basic Research Program (973 Program) of China, 2014CB542400 (to AFC); the National Science Foundation of China, 81370359 and 91339204 (to AFC), 81570271 (to JJC) and 81273594 and 81470535 (to HY), and the National Institutes of Health, R01 GM50441 (to TRB).

\section{CONCLUSION}

Our study established cysteine protease cathepsin $\mathrm{L}$ as a critical regulator of the inflammation that leads to $\mathrm{IH}$ and that the TLR4-MyD88 pathway in myeloid lineages regulates cathepsin L expression in the vessel wall following wire injury. The protease inhibitor SQV blocks $\mathrm{IH}$ though mechanisms that may include the suppression of cathepsin L activity.

\section{DISCLOSURE}

The authors declare that they have no competing interests as defined by Molecular Medicine or other interests that might be perceived to influence the results and discussion reported in this paper.

\section{REFERENCES}

1. Liu MW, Roubin GS, King SB $3^{\text {rd }}$. (1989) Restenosis after coronary angioplasty: potential biologic determinants and role of intimal hyperplasia. Circulation. 79:1374-87.

2. Ross R. (1986) The pathogenesis of atherosclerosis: an update. N. Engl. J. Med. 314:488-500.

3. Welt FG, Rogers C. (2002) Inflammation and restenosis in the stent era. Arterioscler. Thromb. Vasc. Biol. 22:1769-76.

4. Tanaka H, et al. (1993) Sustained activation of vascular cells and leukocytes in the rabbit aorta after balloon injury. Circulation. 88:1788-803.

5. Liu J, Sukhova GK, Sun JS, Xu WH, Libby P, Shi GP. (2004) Lysosomal cysteine proteases in atherosclerosis. Arterioscler. Thromb. Vasc. Biol. 24:1359-66.

6. Creasy BM, McCoy KL. (2011) Cytokines regulate cysteine cathepsins during TLR responses. Cell Immunol. 267:56-66.

7. Sun M, et al. (2011) Cathepsin-L contributes to cardiac repair and remodelling post-infarction. Cardiovasc. Res. 89:374-83.

8. Urbich C, et al. (2005) Cathepsin L is required for endothelial progenitor cell-induced neovascularization. Nat. Med. 11:206-13.

9. Sun J, et al. (2011) Cathepsin L activity is essential to elastase perfusion-induced abdominal aortic aneurysms in mice. Arterioscler. Thromb. Vasc. Biol. 31:2500-08.

10. Bromme D, Li Z, Barnes M, Mehler E. (1999) Human cathepsin V functional expression, tissue distribution, electrostatic surface potential, enzymatic characterization, and chromosomal localization. Biochemistry. 38:2377-85.

11. Santamaria I, et al. (1998) Cathepsin L2, a novel human cysteine proteinase produced by breast and colorectal carcinomas. Cancer Res. 58:1624-30.

12. Cai J, et al. (2015) HMGB1-Driven Inflammation and Intimal Hyperplasia After Arterial Injury Involves Cell-Specific Actions Mediated by TLR4. Arterioscler. Thromb. Vasc. Biol. 35:2579-93.

13. Danenberg HD, et al. (2002) Systemic inflammation induced by lipopolysaccharide increases neointimal formation after balloon and stent injury in rabbits. Circulation. 105:2917-22.

14. Feldman LJ, et al. (2000) Interleukin-10 inhibits intimal hyperplasia after angioplasty or stent implantation in hypercholesterolemic rabbits. Circulation. 101:908-16.

15. Saxena A, et al. (2011) The vascular repair process after injury of the carotid artery is regulated by IL-1RI and MyD88 signalling. Cardiovasc. Res. 91:350-57.

16. Zhang LL, et al. (2011) PPARgamma attenuates intimal hyperplasia by inhibiting TLR4-mediated inflammation in vascular smooth muscle cells. Cardiovasc. Res. 92:484-93.

17. Tang PC, et al. (2008) MyD88-dependent, superoxide-initiated inflammation is necessary for flow-mediated inward remodeling of conduit arteries. J. Exp. Med. 205:3159-71.

18. Buchholz BM, Billiar TR, Bauer AJ. (2010) Dominant role of the MyD88-dependent signaling pathway in mediating early endotoxin-induced murine ileus. Am. J. Physiol. Gastrointest. Liver Physiol. 299:G531-38.

19. Kumar A, Lindner V. (1997) Remodeling with neointima formation in the mouse carotid artery after cessation of blood flow. Arterioscler. Thromb. Vasc. Biol. 17:2238-44.

20. Yu P, Nguyen BT, Tao M, Campagna C, Ozaki CK. (2010) Rationale and practical techniques for mouse models of early vein graft adaptations. J. Vasc. Surg. 52:444-52.

21. Pribis JP, et al. (2015) The HIV protease inhibitor saquinavir inhibits HMGB1 driven inflammation by targeting the interaction of cathepsin $\mathrm{V}$ with TLR4/MyD88. Mol. Med. 5(3):538-46.

22. Chapman HA, Riese RJ, Shi GP. (1997) Emerging roles for cysteine proteases in human biology. Annu. Rev. Physiol. 59:63-88.

23. Cheng XW, Huang Z, Kuzuya M, Okumura K, Murohara T. (2011) Cysteine protease cathepsins in atherosclerosis-based vascular disease and its complications. Hypertension. 58:978-86.

24. Qin Y, Shi GP. (2011) Cysteinyl cathepsins and mast cell proteases in the pathogenesis and therapeutics of cardiovascular diseases. Pharmacol. Ther. 131:338-50.
25. Du X, Chen NL, Wong A, Craik CS, Bromme D. (2013) Elastin degradation by cathepsin V requires two exosites. J. Biol. Chem. 288:34871-81.

26. Cicha I, et al. (2011) Carotid plaque vulnerability: a positive feedback between hemodynamic and biochemical mechanisms. Stroke. 42:3502-10.

27. Li W, Kornmark L, Jonasson L, Forssell C, Yuan XM. (2009) Cathepsin L is significantly associated with apoptosis and plaque destabilization in human atherosclerosis. Atherosclerosis. 202:92-102.

28. Cai J, Wen J, Bauer E, Zhong H, Yuan H, Chen A (2015) The Role of HMGB1 in Cardiovascular Biology: Danger Signals. Antioxid. Redox, Signal. 23(17):1351-69.

29. Akira S, Takeda K, Kaisho T. (2001) Toll-like receptors: critical proteins linking innate and acquired immunity. Nat. Immunol. 2:675-80.

30. Mullick AE, Tobias PS, Curtiss LK. (2006) Toll-like receptors and atherosclerosis: key contributors in disease and health? Immunol. Res. 34:193-209.

31. Arancibia SA, et al. (2007) Toll-like receptors are key participants in innate immune responses. Biol. Res. 40:97-112.

32. Gupta AK, Cerniglia GJ, Mick R, McKenna WG, Muschel RJ. (2005) HIV protease inhibitors block Akt signaling and radiosensitize tumor cells both in vitro and in vivo. Cancer Res. 65:8256-65.

33. Sgadari C, Monini P, Barillari G, Ensoli B. (2003) Use of HIV protease inhibitors to block Kaposi's sarcoma and tumour growth. Lancet Oncol. 4:537-47.

34. Gary-Bobo G, et al. (2010) Effects of HIV protease inhibitors on progression of monocrotaline- and hypoxia-induced pulmonary hypertension in rats. Circulation. 122:1937-47.

35. Coppo R, et al. (2012) Saquinavir in steroiddependent and -resistant nephrotic syndrome: a pilot study. Nephrol. Dial. Transplant. 27:1902-10.

36. Wolf T, Findhammer S, Nolte B, Helm EB, Brodt HR. (2003) Inhibition of TNF-alpha mediated cell death by HIV-1 specific protease inhibitors. Eur. J. Med. Res. 8:17-24.

37. Equils O, Shapiro A, Madak Z, Liu C, Lu D. (2004) Human immunodeficiency virus type 1 protease inhibitors block toll-like receptor 2 (TLR2)- and TLR4-induced NF-kappaB activation. Antimicrob. Agents Chemother. 48:3905-11.

Cite this article as: Cai J, et al. (2017) Cathepsin L promotes vascular intimal hyperplasia after arterial injury. Mol. Med. 23:92-100. 